\title{
Differences in subjective response to alcohol in heavy- and light-drinking Chinese men versus Caucasian American men
}

\author{
Sandra Y. Rueger ${ }^{1,2}$, Hongxing $\mathrm{Hu}^{3,4 *}$, Patrick McNamara', Dingcai $\mathrm{CaO}^{5}$, Wei $\mathrm{Hao}^{3 * *}$ \& \\ Andrea C. King'
}

Department of Psychiatry and Behavioral Neuroscience, The University of Chicago, Chicago, IL, USA, Department of Psychology, Wheaton College, Wheaton, IL, USA, ${ }^{2}$ Mental Health Institute, Second Xiangya Hospital, Central South University, Hunan, China, ${ }^{3}$ Clinical Psychological Department, First Affiliated Hospital, Xinjiang Medical University, Xinjiang, China ${ }^{4}$ and Department of Ophthalmology and Visual Sciences, University of Illinois at Chicago, Chicago, IL, USA ${ }^{5}$

\section{ABSTRACT}

Aims To compare subjective responses to alcohol among Han Chinese and Caucasian American males. Design Double-blinded, placebo-controlled human laboratory design. Participants completed three randomized experimental sessions with high and low alcohol and placebo beverages. Setting Chinese participants were examined at Xinjiang Medical University, China. Caucasian participants were examined at the University of Chicago, USA. Participants Seventy Han Chinese (35 heavy/35 light drinkers) and 75 Caucasian Americans (43 heavy/32 light drinkers). Measurements Breath alcohol concentration (BrAC) and the 'stimulation' and 'sedation' subscales of the Biphasic Alcohol Effects Scale were assessed at pre-drink baseline and four time-points after beverage consumption. The 'like' and 'want' subscales of the Drug Effects Questionnaire were also assessed at the post-drink assessments. Findings Comparisons with light drinkers showed that high- and low-dose alcohol produced decreases in stimulation, liking and wanting in Chinese versus Caucasians $(P<0.05)$, and dose-dependent increases in sedation in both groups $(P<0.001)$. Among heavy drinkers, high-dose alcohol produced higher stimulation $(P<0.001)$ but with concomitant higher sedation for both doses $(P<0.05)$ for Chinese versus Caucasians. Alcohol also demonstrated significantly lower liking $(P<0.001)$ in Chinese versus Caucasian heavy drinkers for both doses. Interestingly, both groups showed dose-dependent increases in wanting relative to placebo $(P<0.05)$, but the magnitude of the increase was lower in Chinese drinkers. Conclusions Stimulating effects of alcohol are predominant in Chinese male binge drinkers, as has been found in Caucasians, but with less hedonic and motivational reward, potentially explaining some of the lower risk for alcohol disorders in Asian subgroups.

Keywords Alcohol, Caucasian, Chinese, ethnicity, heavy drinker, light drinker, reward, sedation, stimulation, subjective response.

Correspondence to: Andrea King, Department of Psychiatry, University of Chicago, 5841 S. Maryland Avenue (MC-3077), Chicago, IL 60637, USA. E-mail: aking@bsd.uchicago.edu

Submitted 13 November 2013; initial review completed 22 January 2014; final version accepted 28 August 2014

\section{INTRODUCTION}

Prominent theories have identified various acute alcohol response phenotypes that may affect risk for alcohol use disorders [1-4]. Recent research has shown that alcohol response factors, including both lower and higher responses, depending on the domain, may play a role in the development of alcohol use disorders (AUDs [4,5]). Work by our group in the United States has shown that greater stimulating, hedonic (liking) and motivational (wanting) responses, as well as lower sedative responses, predicted future drinking problems [5-7]. However, the majority of alcohol challenge research examining acute subjective effects has been conducted using primarily Caucasian participants ([4-11]; see [12,13] for reviews). As drinking levels and associated problems are growing in other racial/ethnic subpopulations, such as those of East Asian origin (e.g. [14,15]), a greater understanding 
of subjective responses to alcohol across several positively and negatively valenced domains is needed to determine whether or not results in Caucasian samples can be extrapolated to other ethnic groups. Examination of the drinking habits of those born and raised in their native country is also important to control for confounding related to the effects of acculturation into American society.

While a commonly held notion in the general population is that Asians are at lower risk for developing AUDs, research has shown that alcohol abuse is a world-wide problem [16,17]. Some Asian subgroups, such as Korean and Japanese men, have demonstrated particularly high rates of alcohol consumption and prevalence of alcohol dependence compared to other Asian subgroups (see $[17,18]$ for reviews). In addition, alcohol consumption in China is rising, with $55 \%$ of men and $15 \%$ of women reporting regular use [19]. Further, life-time prevalence for alcohol use disorders in China is estimated at 9.0\% [20]. While this rate is lower than in primarily Caucasian samples (13-17\% [21]), it is indicative of significant hazardous drinking in a subpopulation of Chinese drinkers.

These increases in alcohol use in China are problematic, in that excessive drinking in this country has been shown to be associated with increased crime [22] and domestic violence [23], traffic accidents [24], alcoholrelated injuries [14] and mortality [16]. There are some cultural factors underlying excessive alcohol use in China, such as peer pressure to drink alcohol and social acceptance of heavy drinking in men [25]. A greater understanding of the predictors of heavy and problematic alcohol use in China is needed to increase our knowledge on the developmental course of AUDs in those of East Asian origin. In particular, it is unknown whether the subjective responses to alcohol shown to predict future problem drinking in primarily Caucasian samples are evident in native Chinese drinkers.

While there are some published reports of alcohol response in people of East Asian origin, they have focused mainly on the genetic deficiency in the aldehyde dehydrogenase (ALHD2) allele and physiological reactions to alcohol. The ALDH2 enzyme deficiency, prevalent in 30-50\% of Asians but $0 \%$ of Caucasians [26,27], has been associated with negative reactions, such as flushing, headache, palpitations, nausea and vomiting ([28-30]; see $[26,27,31]$ for reviews). However, very few studies of people of East Asian origin have focused systematically on subjective effects of alcohol. The majority of studies that address subjective effects of alcohol in Asians or Asian Americans have used retrospective self-reports of alcohol effects (e.g. [32-35]). Others have examined general sedative-like intoxicating effects, such as clumsy or confused, without consideration for concomitant hedonic effects [36-39], or have assessed global effects such as 'feeling terrible (or great) overall' using instruments that are established as reliable and valid measures of alcohol's effects [36-41]. Thus, a direct comparison of alcohol effects using psychometrically sound instruments to assess positive- and negative-like effects in native Chinese and Caucasian Americans is needed to elucidate more clearly if there are ethnic differences in subjective alcohol response [12].

In the present study, we provide the first direct examination of native Chinese versus Caucasian American heavy- and light-drinking men on a range of validated subjective alcohol responses (i.e. stimulation, sedation, liking and wanting) found previously to be related to risk for future drinking and AUD symptoms in primarily Caucasian samples. The Caucasian comparison sample was extracted from our larger prior study [5]. As the majority of heavy drinking in China occurs in men and is relatively rare among women [19], we focused upon male drinkers in this study. The design of this study included administration of two doses of alcohol and a placebo control beverage, with the measurement of multiple subjective effects of alcohol across time-points corresponding to the rising limb, peak, early and later declining limbs. This allowed for a full assessment of subjective alcohol response, which may vary based on the dose administered, the domain measured and the temporal interval from the acute alcohol absorption, peak blood levels and slow elimination phases. Consistent with the literature on negative effects of alcohol experienced by people of Asian descent, we hypothesized that there would be dosedependent differences between Chinese and Caucasian heavy drinkers, with the Chinese having lower alcohol stimulation and reward and higher sedation than Caucasians. Given that light drinkers show primarily sedative responses to alcohol $[5,7]$, we expected both Chinese and Caucasians to show dose-dependent sedative effects with few discernable positive effects.

\section{METHOD}

\section{Participants}

Participants recruited for the study met the following inclusion criteria: age 21-35 years, in good general medical health with no current or past physical or psychiatric disorders and no history of DSM-IV alcohol or substance dependence, including moderate to severe nicotine dependence. The Chinese sample consisted of 70 (35 light/35 heavy drinkers) men of Han Chinese descent living in Xinjiang, China. The Caucasian American group consisted of 75 (32 light/43 heavy drinkers) self-reported Caucasian/white non-Hispanic men extracted from the larger male and female ethnically and racially diverse first wave of the Chicago Social Drinking Project (CSDP) 
sample of 86 light and 104 heavy drinkers [5]. For both the Chinese and Caucasian groups, inclusion criteria for heavy social drinkers were: (i) consuming at least 10 but no more than 40 standard alcoholic drinks per week; and (ii) engaging in regular binge drinking, defined as consuming five or more drinks on any one occasion one to five times on average per week [42]. For light social drinkers, inclusion criteria were: (i) consuming at least one but no more than five standard alcoholic drinks per week; and (ii) engaging in no binge drinking.

\section{Procedures}

The study used a double-blind, placebo-controlled alcohol challenge design, with two within-subject factors (dose and time) and two between-subject factors (ethnicity and drinking group). Experimental conditions were identical between sites, with each subject participating in three sessions and consuming a beverage containing a high dose of alcohol ( $0.8 \mathrm{~g} / \mathrm{kg} ; 16 \%$ volume alcohol), low dose of alcohol $(0.4 \mathrm{~g} / \mathrm{kg} ; 8 \%$ volume alcohol) or placebo beverage $(0.0 \mathrm{~g} / \mathrm{kg} ; 1 \%$ volume of ethanol as taste mask) in random order, with a minimum 48-hour interval between the two sessions. The volume of the high- and low-dose beverage was identical across sessions, with only the alcohol content differing. Given differences in locally available ethanol and mixers, for Chinese the beverage consisted of 52-proof alcohol mixed with iced tea beverage and for Caucasians the 95-proof alcohol was mixed with water, flavored drink mix and a sucralosebased sugar substitute. To minimize expectancy bias, the participants were told that they would consume a beverage containing a sedative, stimulant, alcohol or placebo (no drug/alcohol).

Participants were asked to abstain from alcohol for 24 hours and cigarette smoking for 3 hours before and for 5 hours during each of the three sessions. Upon arrival, participants underwent a breathalyzer test to confirm compliance with alcohol abstinence instructions [breath alcohol concentration $(\mathrm{BrAC})<0.003]$. They also completed baseline measures of subjective measures (i.e. stimulation and sedation). After 30 minutes, the participants consumed the beverage, divided into two equal parts, within 15 minutes in the experimenter's presence. They then completed subjective measures (i.e. stimulation, sedation, liking and wanting) at 30, 60, 120 and 180 minutes after initiation of the beverage to approximate the rising limb, peak BrAC, early and late declining limbs, respectively. The data collections were approved at the respective Institutional Review Boards for each site.

\section{Measures}

Study instructions and measures were given in the participants' native language, i.e. Mandarin Chinese and
English. For Mandarin Chinese, the measures were translated and back-translated by two independent bilingual staff.

\section{Biphasic Alcohol Effects Scale (BAES [43])}

The BAES is a reliable and valid measure of biphasic subjective response to alcohol [44]. The questionnaire consists of seven items that assess stimulant response and seven items that assess sedative response on an 11-point rating scale, ranging from not at all (0) to extremely (10). The alphas for the current Chinese/Caucasian samples for stimulation ranged, respectively, from 0.88 to 0.98 / 0.93 to 0.97 for heavy drinkers and 0.87 to $0.97 / 0.88$ to 0.96 for light drinkers, and for sedation ranged from 0.77 to $0.85 / 0.87$ to 0.96 for heavy drinkers and 0.77 to $0.87 / 0.81$ to 0.96 for light drinkers.

\section{Drug effects questionnaire (DEQ [45])}

The DEQ is a reliable and valid instrument consisting of several adjectival items of drug effects using 100-mm visual analog scales. The two items used in the current study assessed alcohol liking ('Do you LIKE the effects you are feeling right now') and wanting ('Would you like MORE of what you consumed right now'). Liking was rated from $0=$ dislike to $100=$ like very much, with midpoint indicating neutral. Wanting was rated from $0=$ not at all to $100=$ very much, with mid-point indicating moderate levels of wanting.

\section{BrAC}

BrAC was collected 30 minutes prior to beverage consumption, as well as 30, 60, 120 and 180 minutes after consumption using the CA2000 Digital Alcohol Detector (South Korea) for the Chinese sample and the AlcoSensor IV, Intoximeter (St Louis, MO, USA) for the Caucasian sample. Approximately $7 \%$ of the BrAC values were $\geq 2.0$ standard deviations (SD) above the mean during the rising limb to peak BrAC. This was due to experimenter error, i.e. not having participants rinse with a small amount of water prior to the first reading. As such, these outlier data points were excluded in BrAC analysis.

\section{Data analytical strategy}

Subjective alcohol responses were analyzed with generalized estimation equation models (GEE) to account for correlations among repeated measures. GEE analyses were conducted separately for light and heavy drinkers to examine the effects of ethnicity (Chinese and Caucasian), dose (high, low and placebo) and post-beverage time [30 minutes (rising limb of BrAC), 60 minutes (peak BrAC), 120 minutes and 180 minutes (early and later declining 
Table 1 Means and standard deviations of light and heavy drinkers of Caucasian American or Chinese ethnicity.

\begin{tabular}{|c|c|c|c|c|c|c|}
\hline & \multicolumn{3}{|c|}{ Light drinkers $(n=67)$} & \multicolumn{3}{|c|}{ Heavy drinkers $(n=78)$} \\
\hline & $\mathrm{CH}$ & $C A$ & t-test & $\mathrm{CH}$ & $C A$ & t-test \\
\hline Age (years) & $23.7(4.0)$ & $26.0(3.4)$ & $2.51^{*}$ & $25.6(4.0)$ & $25.0(3.0)$ & -0.76 \\
\hline Education & $14.9(2.6)$ & $17.2(2.2)$ & $3.71^{*}$ & $15.1(3.0)$ & $16.0(1.5)$ & 1.54 \\
\hline Age of first drink & $16.1(2.5)$ & $17.3(2.9)$ & 1.99 & $16.1(2.7)$ & $14.7(2.8)$ & $-2.39^{*}$ \\
\hline AUDIT & $3.2(0.7)$ & $3.3(1.0)$ & 0.80 & $9.8(2.2)$ & $11.9(3.2)$ & $3.38^{* *}$ \\
\hline Drink days/month & $6.5(2.0)$ & $7.0(3.6)$ & 0.61 & $13.0(2.9)$ & $14.2(5.4)$ & 1.18 \\
\hline Drinks/drinking day & $2.4(0.7)$ & $1.8(0.6)$ & $-4.00^{* *}$ & $6.3(1.4)$ & $5.6(2.0)$ & -1.75 \\
\hline Binges/month & - & - & - & $6.8(2.1)$ & $7.9(2.8)$ & 1.90 \\
\hline Cigarettes per day & $5.3(3.2)$ & $5.3(5.5)$ & -0.01 & $9.4(4.3)$ & $6.5(5.2)$ & -1.97 \\
\hline
\end{tabular}

AUDIT $=$ Alcohol Use Disorders Identification Test; $\mathrm{CH}=$ Han Chinese; $\mathrm{CA}=$ Caucasian American. Cigarettes per day are for current smokers: $\mathrm{CH}$ light drinkers $(n=16)$, US light drinkers $(n=4)$, CH heavy drinkers $(n=18)$, US heavy drinkers $(n=29)$. ${ }^{*} P<0.05$; ${ }^{* *} P<0.01$.

limb of $\mathrm{BrAC})]$ and their interactions. For stimulation and sedation, the pre-drink baseline measure was included as a covariate, but not for liking and wanting, as there was no pre-drink baseline measure. For each outcome, the GEE model with the three-way and all the two-way interaction terms and the main effects was examined first. If the three-way interaction was not significant, then the model was repeated after removing this interaction term. Subsequent post-estimation comparisons were limited to a significant ethnicity $\times$ dose $\times$ time interaction, as well as the ethnicity $\times$ dose interaction and main effect of ethnicity. All analyses were conducted in Stata version 12.0.

\section{RESULTS}

\section{Background characteristics}

A comparison of general demographic and alcohol drinking characteristics between Chinese and Caucasian light and heavy social drinkers are reported in Table 1 . The groups were largely similar on most characteristics, with the exception that Chinese versus Caucasian heavy drinkers started drinking at an older age and had slightly lower Alcohol Use Disorder Identification Test (AUDIT) scores. Among light drinkers, Chinese were younger, had fewer years of education and reported 0.6 more drinks on average per drinking occasion. Further, smoking prevalence was higher in Chinese versus Caucasian light drinkers (46 versus 13\% current smokers, respectively, $P<0.01$ ), but smoking was relatively light, with both groups averaging 5.3 cigarettes per day. The heavydrinking Chinese and Caucasian groups did not differ on smoking status (51 versus $67 \%$, respectively) or cigarettes per day (9.4 versus 6.5, respectively). Smoking status was not correlated with any subjective effects.

\section{BrAC levels and associations with subjective effects}

Post-drink BrAC at low and high doses was compared between ethnic groups, and there was a significant three-
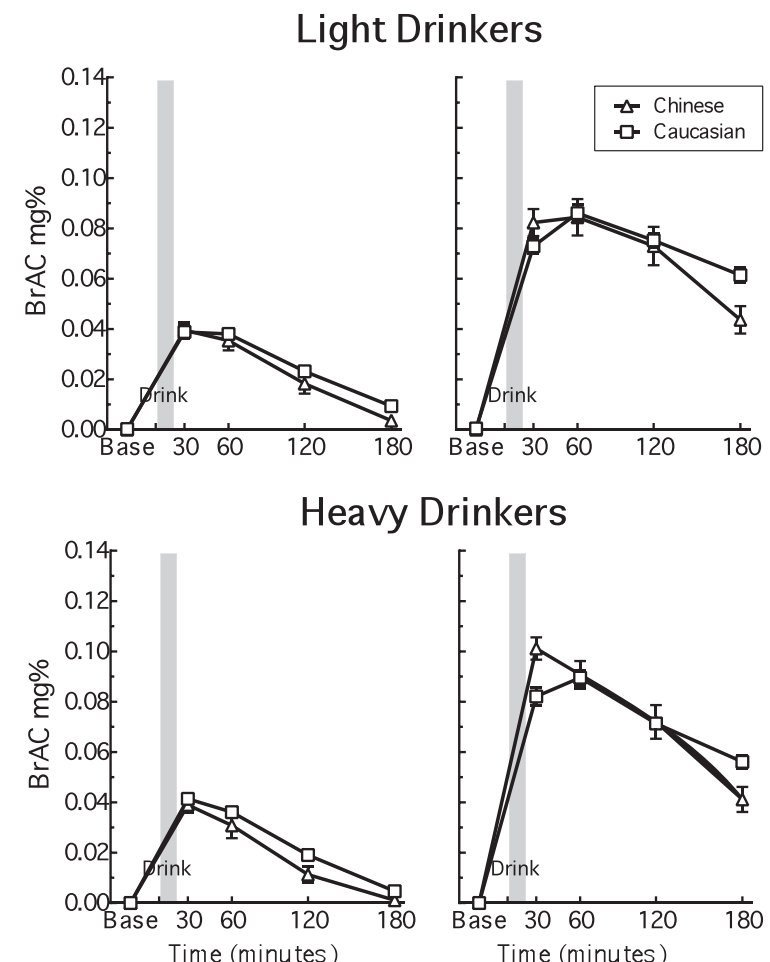

Low Alcohol Dose

High Alcohol Dose

Figure I Breath alcohol concentration (BrAC). These graphs show the $\mathrm{BrAC}$ during the rising and declining limbs of the $\mathrm{Br} A C$ curve of light and heavy drinkers at low and high doses of alcohol

way interaction for both heavy $\{\beta[$ standard error $(\mathrm{SE})]=-0.011(0.002), P<0.001\}$ and light drinkers $[\beta(\mathrm{SE})=-0.009$ (0.003), $P<0.01]$ (see Fig. 1). At the high but not low alcohol dose, the Chinese group, compared with the Caucasian group, had significantly higher BrAC at the rising limb $(P<0.01$ for light drinkers; $P=0.001$ for heavy drinkers) with lower BrAC at the later declining limb $(P=0.001$ for light drinkers; $P<0.001$ for heavy drinkers). Examination of biphasic 
Table 2 Results of GEE analyses for heavy and light drinkers focused on ethnicity.

\begin{tabular}{|c|c|c|c|c|c|c|}
\hline \multirow[b]{2}{*}{ Subjective response } & \multicolumn{3}{|c|}{ Light drinkers $(n=67)$} & \multicolumn{3}{|c|}{ Heavy drinkers $(n=78)$} \\
\hline & $\beta$ & $S E$ & $P$ & $\beta$ & $S E$ & $P$ \\
\hline \multicolumn{7}{|l|}{ Stimulation } \\
\hline Ethnicity & $-3.233^{\mathrm{a}}$ & 1.908 & 0.090 & $-1.491^{\mathrm{a}}$ & 1.966 & 0.448 \\
\hline Ethnicity $\times$ dose $^{\mathrm{a}}$ & $-2.241^{\mathrm{a}}$ & 0.627 & 0.006 & $3.028^{\mathrm{a}}$ & 0.654 & $<0.001$ \\
\hline Ethnicity $\times$ dose $\times$ time $^{a}$ & 0.963 & 0.560 & 0.085 & 1.022 & 0.580 & 0.077 \\
\hline \multicolumn{7}{|l|}{ Sedation } \\
\hline Ethnicity & $3.327^{\mathrm{a}}$ & 2.480 & 0.180 & -5.983 & 2.473 & 0.016 \\
\hline Ethnicity $\times$ dose & $-1.848^{\mathrm{a}}$ & 0.807 & 0.022 & 6.442 & 1.494 & $<0.001$ \\
\hline Ethnicity $\times$ dose $\times$ time & -.465 & 0.716 & 0.516 & -1.396 & 0.544 & 0.010 \\
\hline \multicolumn{7}{|l|}{ Like } \\
\hline Ethnicity & $-17.557^{\mathrm{a}}$ & 4.812 & $<0.001$ & -14.225 & 5.023 & 0.005 \\
\hline Ethnicity $\times$ dose & $-5.360^{\mathrm{a}}$ & 1.731 & 0.002 & -16.661 & 3.296 & $<0.001$ \\
\hline Ethnicity $\times$ dose $\times$ time & 1.625 & 1.548 & 0.294 & 2.735 & 1.204 & 0.023 \\
\hline \multicolumn{7}{|l|}{ Want more } \\
\hline Ethnicity & $-9.232^{\mathrm{a}}$ & 4.677 & 0.048 & $-21.750^{\mathrm{a}}$ & 5.142 & 0.004 \\
\hline Ethnicity $\times$ dose & $-1.108^{\mathrm{a}}$ & 1.550 & 0.475 & $-3.234^{\mathrm{a}}$ & 1.562 & 0.038 \\
\hline Ethnicity $\times$ dose $\times$ time & 0.117 & 1.386 & 0.932 & 1.849 & 1.396 & 0.185 \\
\hline
\end{tabular}

${ }^{a}$ Values are taken from analyses with the non-significant three-way interaction removed. GEE = generalized estimation equation models; SE $=$ standard error.

BrAC levels and subjective responses for both groups and both doses, however, showed no correlations of BrAC level at the rising limb with stimulation, liking or wanting ratings or BrAC level at the declining limb with sedation ratings. Finally, the subjective response to placebo dose was compared between Chinese and Caucasian drinkers and there were no significant differences in either heavyor light-drinking groups.

\section{Ethnic differences in alcohol effects among light drinkers}

None of the three-way interactions of ethnicity, dose and time were significant for light drinkers (Table 2; Fig. 2). The subsequent GEE models revealed significant ethnicity $\times$ dose interactions for stimulation, sedation and like (Table 2). More specifically, at both high and low alcohol doses, alcohol produced lower stimulation in the Chinese versus Caucasian group $(P<0.01)$. Alcohol produced dose-dependent increases in sedation for both ethnic groups (high dose $>$ low dose $>$ placebo, $P<0.001$ ). Both doses of alcohol reduced liking ratings (versus placebo) for the Chinese $(P<0.001)$, with liking at both doses significantly lower in the Chinese versus the Caucasians $(P<$ 0.05 for low dose; $P<0.01$ for high dose). Finally, there was a significant main effect of wanting, with the Chinese group reporting lower ratings for wanting than Caucasians, but this was not specific to beverage condition.

\section{Ethnic differences in alcohol effects among heavy drinkers}

The three-way interaction of ethnicity, dose and time was significant for sedation and liking, but not for stimulation and wanting (Table 2; Fig. 3). For sedation, postestimation analyses for the three-way interaction showed that two effects accounted for the significant interaction: high-dose alcohol increased sedation more in the Chinese compared with the Caucasian groups during the rising limb $(P=0.05)$ and low-dose alcohol increased sedation more in the Chinese compared with the Caucasian groups at peak $\operatorname{BrAC}(P=0.01)$ and early declining limb $(P<0.01)$. For liking, post-estimation analyses for the three-way interaction showed that both alcohol doses produced lower liking in the Chinese compared with the Caucasian groups at rising limb and peak $\operatorname{BrAC}(P<0.001)$. For stimulation, there was a significant ethnicity $\times$ dose interaction (Table 2) such that high-dose alcohol produced significantly greater stimulation in Chinese compared with Caucasian heavy drinkers $(P<0.001)$. For wanting, the significant ethnicity $\times$ dose interaction showed that both alcohol doses produced lower wanting in Chinese versus Caucasians $(P<0.001)$, with each group showing dose-dependent increases in wanting relative to placebo (high $>$ low $>$ placebo, $P<0.05$ ).

\section{DISCUSSION}

The current study investigated subjective responses to alcohol in light- and heavy-drinking Han Chinese and Caucasian American males, and the results supported hypotheses about dose-dependent differences between the two cultural groups. Chinese light drinkers reported lower stimulation, liking and wanting alcohol at both doses than their Caucasian counterparts, but similar 


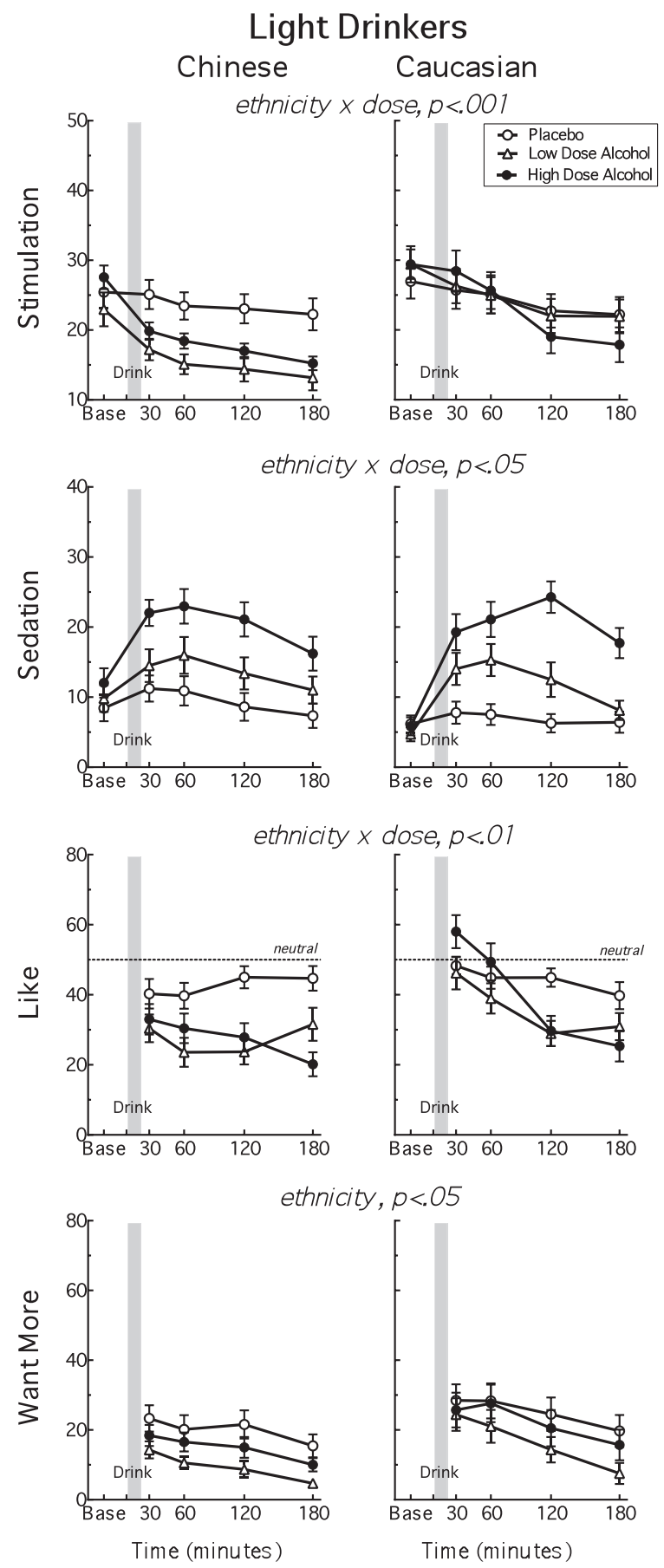

Figure 2 Han Chinese versus Caucasian American light drinkers. These graphs show the acute response to high and low doses of alcohol and placebo in Chinese versus Caucasian American light drinkers across the breath alcohol concentration $(\mathrm{BrAC})$ curve

increases in sedation. In addition, Chinese heavy drinkers showed higher sedation and less liking (hedonic reward) during the alcohol absorption phase (i.e. rising limb), and a lower overall magnitude of wanting (motivational reward) alcohol than in Caucasians. However, an unexpected finding showed that Chinese heavy drinkers also showed higher sensitivity to the stimulating effects of the

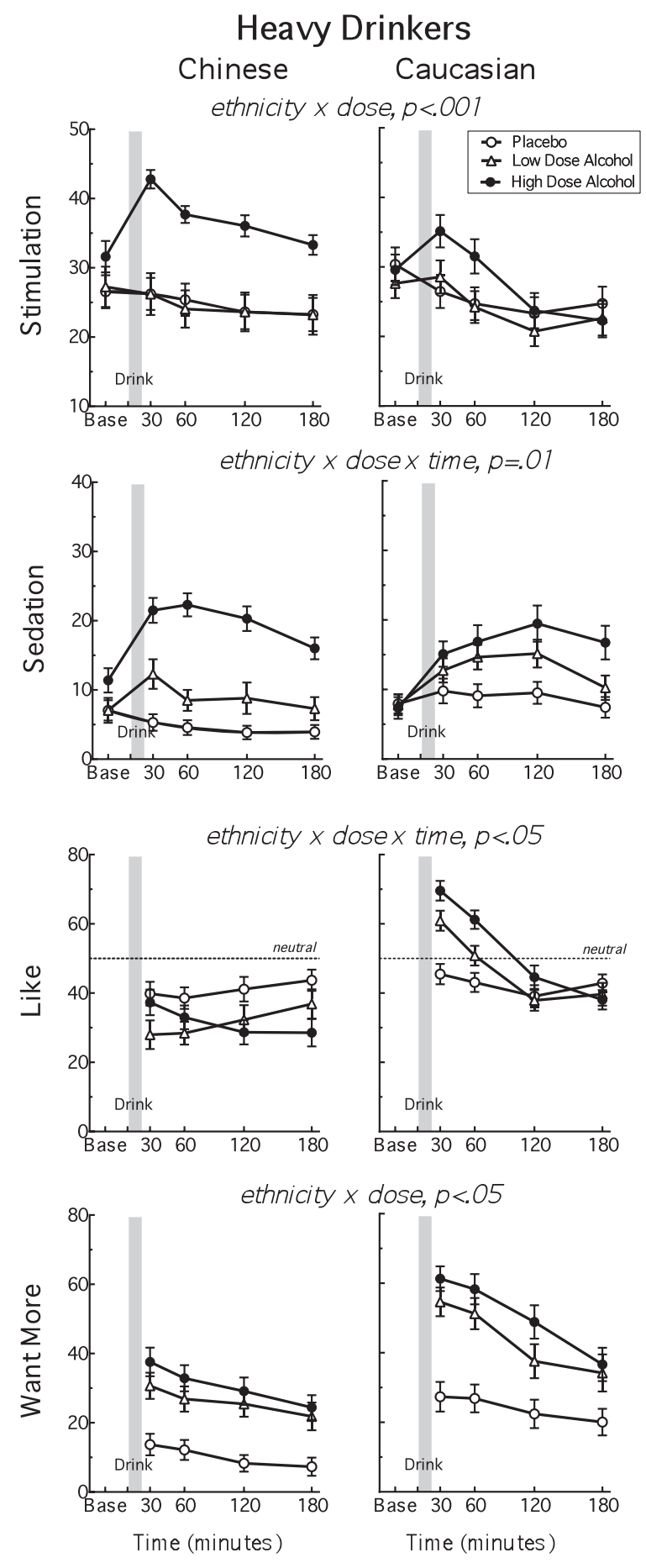

Figure 3 Han Chinese versus Caucasian American heavy drinkers. These graphs show the acute response to high and low doses of alcohol and placebo in Chinese versus Caucasian American heavy drinkers across the breath alcohol concentration (BrAC)curve

high alcohol dose than their Caucasian counterparts. This is unlikely to be due to metabolism, as while Chinese drinkers had higher BrAC levels during the rising limb compared with Caucasian drinkers, the differences were relatively small and $\mathrm{BrAC}$ was not associated with subjective effects. 
These results suggest that Chinese social drinkers experience fewer of the hedonic effects of alcohol relative to their Caucasian counterparts, regardless of dose of alcohol or drinking history. Importantly, despite the fact that heavy-drinking Chinese men were more sensitive to alcohol's stimulant effects, this response was not accompanied by hedonic reward (liking). In addition, their relatively high levels of concomitant sedation might have mitigated the observed stimulatory effects, while remaining in motivational salience (i.e. wanting more) in heavy drinkers.

The lower alcohol liking and relative lesser magnitude of wanting for Chinese heavy drinkers compared to their Caucasian counterparts suggests that factors beyond subjective effects of alcohol may promote alcohol consumption and/or the development of risky drinking habits in Asian men. While not assessed in this laboratory-based study, there may be differential external cultural and social influences to drink alcohol or engage in binge drinking, such as the social acceptance of heavy drinking in Chinese men [41]. These results suggest that clinical interventions for alcohol use in Chinese men should consider inclusion of the cultural/social influences and motives to drink alcohol and not focus solely on the role of pleasurable and hedonic effects, which have been found in a pilot study by our group in the United States to improve outcomes when added to standard brief intervention [46].

Strengths of this study include the use of a mixedsubject design, direct measures of subjective alcohol response and examination of two doses of alcohol as well as placebo across multiple time-points for a full examination of alcohol response throughout the BrAC. This is important, because there is no ubiquitous response to alcohol, and clarifying differential responses based on the dose, the domain measured and effects throughout alcohol absorption, peak breath levels and the slow elimination phases are essential. Another strength was the use of similar methodologies in China and the United States to facilitate direct ethnic comparisons, and provision of the subjective scales in the native language.

There are also some limitations in this study that suggest future directions for research. First, genetic data were not obtained and therefore effects of the $A L D H$ and other genetic polymorphisms were not ascertained. Anecdotal observational reports of the participants in the current Chinese sample indicated that more than half showed some degree of facial flushing, consistent with the effects associated with the ALDH2 deficiency reported in population-based estimates [26]; however, inclusion of genetic data could have added important information about possible genetic underpinnings of the reported subject responses. Interestingly, studies with
East Asian samples have demonstrated that ALDH2 deficiency does not offer complete protection against alcoholism [33,47], as suggested by reports of positive response (e.g. 'great' feelings) to alcohol shown in those with ALDH2 deficiency [36,40,41]. Thus, more studies that include assessment of genetic polymorphisms of alcohol metabolizing alleles are needed to elucidate the ways in which this biological factor is related, independently or interactively, to pleasurable and rewarding effects of alcohol as well as sedating and noxious effects. However, caution has been advised in undertaking studies focused on candidate genes without sufficient sample size [48], and alcohol response is probably a multi-factorial and polygenic phenomenon. Thus, future studies that include genetic data should do so with enough power to offer robust findings on genotypephenotype interactions.

Secondly, only men were examined in this study, so it is unclear whether the same responses would be found in women. Binge drinking in Chinese women remains rare [19], thus recruiting them would have been challenging. Thirdly, the study did not include contextual factors involved in alcohol use, and it is possible that Chinese heavy-drinking men may have different motives for engaging in frequent binge drinking than their Caucasian American counterparts. Finally, the current study focused on light to moderate smokers, and thus factors related to smoking-drinking co-use were not ascertained. Given the high percentage of Chinese who smoke cigarettes, future consideration of the role that smoking may have on subjective effects of alcohol would add to our understanding of heavy and risky alcohol use patterns in Chinese drinkers [49].

In summary, this is the first study of its kind to offer native Chinese versus Caucasian American cross-ethnic comparisons on alcohol use and subjective response to alcohol in people with heavy- and light-drinking phenotypes. The results of this study suggest that Chinese male heavy drinkers show a differential pattern of response to alcohol than their Caucasian American counterparts. While both groups demonstrate stronger stimulant effects than in their light-drinker controls, for Chinese men overall alcohol was experienced as more sedating and less pleasurable than in Caucasians. In spite of this, the heavy-drinking Chinese men engaged in binge drinking at levels similar to their Caucasian counterparts. Importantly, assumptions about subjective effects as risk factors based on Caucasian samples that have guided previous research may not apply to other cultures and racial/ethnic groups, especially those of East Asian origin. Future research is warranted to discern factors involved in acute alcohol response differences in people of East Asian origin from Caucasians and other ethnic subgroups. 


\section{Declaration of interests}

None.

\section{Acknowledgements}

This research was supported by NIH/NIAAA Research Supplement to Promote Diversity in Health-Related Research no. R01-AA013746-S1 (S.R.), NIH/NIAAA no. R01-AA013746 (A.K.), NIH UL1RR024999 ITM/CTSA award (A.K.) and ABMRF/Foundation for Alcohol Research (DC) in the United States, and the State Key Program of the National Natural Science of China Grant no. 81130020 (W.H.), the National 12th Five Year Plan Support Project Grant no. 2012BAI01B07 (W.H.) and the National Key Basic Research and Development Program Grant no. 2009CB522007 (W.H.) in China.

\section{References}

1. Newlin D. B., Thomson J. B. Alcohol challenge with sons of alcoholics: a critical review and analysis. Psychol Bull 1990; 108: 383-402.

2. Robinson T. E., Berridge K. C. The neural basis of drug craving: an incentive-sensitization theory of addiction. Brain Res Rev 1993; 18: 247-91.

3. Robinson T. E., Berridge K. C. Incentive-sensitization and addiction. Addiction 2001; 96: 103-14.

4. Schuckit M. A. Low level of response to alcohol as a predictor of future alcoholism. Am J Psychiatry 1994; 151: 184-9.

5. King A. C., de Wit H., McNamara P. J., Cao D. Rewarding, stimulant, and sedative alcohol responses and relationship to future binge drinking. Arch Gen Psychiatry 2011; 68: 389-99.

6. King A. C., McNamara P. J., Hasin S., Cao D. C. Alcohol challenge responses predict future alcohol use disorder syndromes: a 6-year prospective study. Biol Psychiatry; 2014; 75: 798-806.

7. King A. C., Houle T., de Wit H., Holdstock L., Schuster A. Biphasic alcohol response differs in heavy versus light drinkers. Alcohol Clin Exp Res 2002; 26: 827-35.

8. Rose A. K., Grunsell L. The subjective, rather than the disinhibiting, effects of alcohol are related to binge drinking. Alcohol Clin Exp Res 2008; 32: 1096-104.

9. Wetherill L., Morzarati S. L., Foroud T., Windisch K., Darlington T., Zimmerman U. S. et al. Subjective perceptions associated with the ascending and descending slopes of breath alcohol exposure vary with recent drinking history. Alcohol Clin Exp Res 2012; 36: 1050-7.

10. Rose A. K., Duka T. Effects of alcohol on inhibitory processes. Behav Pharmacol 2008; 19: 284-91.

11. Zimmerman U. S., Mick I., Laucht M., Vitvitskiy V., Plawecki M. H., Mann K. F. et al. Offspring of parents with an alcohol use disorder prefer higher levels of brain alcohol exposure in experiments involving computer-assisted self-infusion of ethanol (CASE). Psychopharmacology 2009; 202: 689-97.

12. Quinn P. D., Fromme K. Subjective response to alcohol challenge: a quantitative review. Alcohol Clin Exp Res 2011; 35 : $1-12$.
13. Ulrich S., Zimmermann U. S., O'Connor S., Ramchandani V. A. Modeling alcohol self-administration in the Human Laboratory. Curr Top Behav Neurosci 2013; 13: 315-53. doi: 10.1007/7854_2011_149.

14. Cherpitel C. J., Ye Y., Bond J., Borges G., Chou P., Nilsen P. et al. Multi-level analysis of alcohol-related injury and drinking patterns: emergency department data from 19 countries. Addiction 2012; 107: 1263-72.

15. Tang Y., Xiang X., Wang X., Cubells J. F., Babor T. F., Hao W. Alcohol and alcohol-related harm in China: policy changes needed. Bulletin of World Health Organization. Public Health Rev 2013; 91: 270-6.

16. World Health Organization. Global status report on alcohol and health [internet]. 2010. Available at: http://www.who .int/substance_abuse/publications/global_alcohol_report/ msbgsruprofiles.pdf (accessed 26 September 2014) (Archived at http://www.webcitation.org/6SrrlPHA1 25 September 2014).

17. Chen C. C., Yin S. J. Alcohol abuse and related factors in Asia. Int Rev Psychiatry 2008; 20: 425-33.

18. Makimoto K. Drinking patterns and drinking problems among Asian-Americans and Pacific Islanders. Alcohol Health Res World 1998; 27: 270-5.

19. Li Y., Jiang Y., Zhang M., Yin P., Wu F., Zhao W. Drinking behaviour among men and women in China: the 2007 China Chronic Disease and Risk Factor Surveillance. Addiction 2011; 106: 1946-56.

20. Phillips M. R., Zhang J., Shi Q., Song Z., Ding Z., Pang S. et al. Prevalence, treatment, and associated disability of mental disorders in four provinces in China during 200105: an epidemiological survey. Lancet 2009; 373: 204153.

21. Hasin D. S., Stinson F. S., Ogburn E., Grant B. F. Prevalence, correlates, disability, and comorbidity of DSM-IV alcohol abuse and dependence in the United States: results from the National Epidemiologic Survey on Alcohol and Related Conditions. Arch Gen Psychiatry 2007; 64: 830-42.

22. Zhang D. J., Lin Y., Mao Y. J., Hu Z. Q. A study on characteristics of criminals with alcohol-related offence in forensic psychiatry. J Forensic Med 2003; 23: 86-9.

23. Zeng H., Wu X. Y. Psychosocial factors associated with domestic violence. Chin J Dis Contr Prevent 2010; 14: 104952 .

24. Zhang W., Zhao J., Yu J., Wu F., Liu W., Zhou Z. et al. Alcohol and other contributing factors among drivers who died in traffic accidents. Chin J Forensic Med 2007; 22: 120-3.

25. Zhou L., Zhang G. B., Hu H. X., Fan Z. J., Hao W. Perceived interpersonal pressure and drinking behavior in South China. Drug Alcohol Depend 2013; 130: 122-8.

26. Eng M. Y., Luczak S. E., Wall T. L. ALDH2, ADH1B, and ADH1C genotypes in Asians: a literature review. Alcohol Res Health 2007; 30: 22-7.

27. Luczak S. E., Glatt S. J., Wall T. L. Meta-analyses of ALDH2 and ADH1B with alcohol dependence in Asians. Psychol Bull 2006; 132: 607-21.

28. Takeshita T., Morimoto K. Self-reported alcohol-associated symptoms and drinking behavior in three ALDH2 genotypes among Japanese university students. Alcohol Clin Exp Res 1999; 23: 1065-9.

29. Obata A., Morimoto K., Sato H., Takeshita T., Kawaguchi H., Koizumi H. et al. Effects of alcohol on hemodynamic and cardiovascular reaction in different genotypes. Psychiatry Res 2005; 139: 65-72. 
30. Nishimura F. T., Kimura Y., Abe S., Fukunaga T., Minami J., Tanii H. et al. Effects of functional polymorphisms related to catecholaminergic systems on changes in blood catecholamine and cardiovascular measures after alcohol ingestion in the Japanese population. Alcohol Clin Exp Res 2008; 32: 1937-46.

31. Ducci F., Goldman D. The genetic basis of addictive disorders. Psychiatr Clin North Am 2012; 35: 495-519.

32. Duranceaux N. C. E., Shuckit M. A., Luczak S. E., Eng M. Y., Carr L. G., Wall T. L. Ethnic differences in level of response to alcohol between Chinese Americans and Korean Americans. J Stud Alcohol Drugs 2008; 69: 227-34.

33. Hendershot C. S., Collins S. E., George W. H., Wall T. L., McCarthy D. M., Liang T. et al. Associations of ALDH2 and ADH1B genotypes with alcohol-related phenotypes in Asian young adults. Alcohol Clin Exp Res 2009; 33: 83947.

34. Luczak S. E., Pandika D., Shea S. H., Eng M. Y., Liang T., Wall T. L. ALDH2 and ALDH1B interactions in retrospective reports of low-dose reactions and initial sensitivity to alcohol in Asian American college students. Alcohol Clin Exp Res 2011; 35: 1238-45.

35. Yokoyama M., Yokoyama A., Yokoyama T., Funazu K., Hamana G., Kondo S. et al. Hangover susceptibility in relation to aldehyde dehydrogenase-2 genotype, alcohol flushing, and mean corpuscular volume in Japanese workers. Alcohol Clin Exp Res 2005; 29: 1165-71.

36. Cook T. A. R., Luczak S. E., Shea S. H., Ehlers C. L., Carr L. G., Wall T. L. Associations of ALDH2 and ADH1B genotypes with response to alcohol in Asian Americans. J Stud Alcohol 2005; 66: 196-204.

37. Chen Y., Peng G., Tsao T., Wang M., Lu R., Yin S. Pharmacokinetic and pharmacodynamics basis for overcoming acetaldehyde-induced adverse reaction in Asian alcoholics, heterozygous for the variant $\mathrm{ALDH} 2 * 2$ gene allele. Pharmacogenet Genomics 2009; 19: 588-99.

38. Peng G., Wang M., Chen C., Luu S., Chou H., Li T. et al. Involvement of acetaldehyde for full protection against alcoholism by homozygosity of the variant allele of mitochondrial aldehyde dehydrogenase gene in Asians. Pharmacogenetics 1999; 9: 463-76.
39. Luczak S. E., Elvine-Kreis B., Shea S. H., Shoshana H., Carr L. G., Wall T. L. Genetic risk for alcoholism relates to level of response to alcohol in Asian-American men and women. J Stud Alcohol 2002; 63: 74-82.

40. Chen Y. C., Lu R. B., Peng G. S., Wang M. F., Wang H. K., Ko H. C. et al. Alcohol metabolism and cardiovascular response in an alcoholic patient homozygous for the ALDH2*2 variant gene allele. Alcohol Clin Exp Res 1999; 23: 185360.

41. Wall T. L., Thomasson H. R., Schuckit M. A., Ehlers C. L. Subjective feelings of alcohol intoxication in Asians with genetic variations of ALDH2 alleles. Alcohol Clin Exp Res 1992; 16: 991-5.

42. Substance Abuse and Mental Health Services Administration (SAMHSA). National Survey on Drug Use and Health. Rockville, MD: Office of Applied Studies; 2005.

43. Martin C. S., Earlywine M., Musty R. E., Perrine M. W., Swift R. M. Development and validation of the Biphasic Alcohol Effects Scale. Alcohol Clin Exp Res 1993; 17: 140-6.

44. Rueger S. Y., McNamara P. J., King A. C. Expanding the utility of the Biphasic Alcohol Effects Scale (BAES) and initial psychometric support for the Brief-BAES (B-BAES). Alcohol Clin Exp Res 2009; 33: 1-9.

45. Morean M. E., Corbin W. R., Treat T. A. The Anticipated Effects of Alcohol Scale: development and psychometric evaluation of a novel assessment tool for measuring alcohol expectancies. Psychol Assess 2012; 24: 1008-23.

46. Fridberg D., King A. Integrating alcohol challenge feedback in a brief intervention for young adult heavy-drinking smokers: A pilot study. Alcohol Clin Exp Res 2014; 38(S1): 211A.

47. Quertemont E. Genetic polymorphism in ethanol metabolism: acetaldehdyde contribution to alcohol abuse and alcoholism. Mol Psychiatry 2004; 9: 570-81.

48. Hart A. B., de Wit H., Palmer A. A. Candidate gene studies of a promising intermediate phenotype: failure to replicate. Neuropsychopharmacology 2013; 38: 802-16.

49. Wu Z. G., Su Z. H., Hao W. Demographic characteristics and related factors of alcohol dependence in five areas in China. Chin J Behav Med Sci 2004; 13: 52-4. 